\section{Edyeasáa

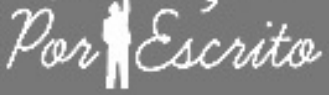

ARTIGO

\section{Editor}

Alexandre Anselmo Guilherme PUCRS, RS, Brasil

\section{Editor Assistente}

Cibele Cheron

PUCRS, RS, Brasi

\section{Editores Associados}

Bruno Antonio Picoli

Universidade Federal da Fronteira Sul, Chapecó, SC, Brasil

Pricila Kohls dos Santos Universidade Católica de Brasília, Brasília, DF, Brasil

Renato de Oliveira Brito

Universidade Católica de Brasilia, Brasilia, DF, Brasil

Elisa Ustarroz

PUCRS, Porto Alegre, RS, Brasil

\section{ISSN 2179-8435}

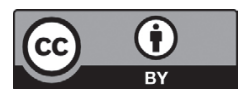

Este artigo está licenciado sob forma de uma licença Creative Commons Atribuição 4.0 Internacional, que permite uso irrestrito, distribuicăo e reprodução seja corretamente citada

\title{
Alteridade, ética e diversidades de gênero na (trans)formação de operadores do Direito e da cultura jurídica
}

\author{
Otherness, ethnic and gender diversity in the (trans)formation
} of Law operators and juridical culture

\author{
Camila Paola Fernandes Polonia $\odot^{1}$ \\ Cibele Cheron $~^{2}{ }^{2}$ \\ ${ }^{1}$ Centro Universitário Ritter dos Reis (UniRitter), Porto Alegre, Brasil.
} 2Pontifícia Universidade Católica do Rio Grande do Sul (PUCRS), Porto Alegre, RS, Brasil.

\section{RESUMO}

O presente artigo traz ao debate a importância de as questões inerentes às diversidades de gênero estarem contempladas na formação dos operadores do Direito. Partimos da constatação do exaurimento da cultura jurídica centrada nos valores e nas práticas adversariais de maximização do ganho individualista e desumanização das relações sócio-políticas. O estudo filia-se ao entendimento de que diferentes saberes estão relacionados a diferentes costumes, condutas e práxis. Assim, transformações na matriz de conhecimentos que formam cidadãos têm potencial para produzir transformações nas relações sociais. Adota-se referencial teórico interdisciplinar com aportes das Ciências Sociais, do Direito, da Educação e da Filosofia a fim de avaliar a inserção de temas relativos às diversidades de gênero como obrigatórios nos projetos pedagógicos e nos currículos de cursos de graduação em Direito de Porto Alegre/RS e região, conforme a Política de Educação em Direitos Humanos vigente. Destacam-se os conceitos de conflito, política, ética e alteridade, em Jacques Rancière, liberalismo, racionalidade política, lutas transversais e saber-poder em Michel Foucault para sustentar a necessária transformação da cultura jurídica e da formação acadêmica. Afirma-se o potencial individual não como um bem em si, mas para que se promova a integração a partir das diferenças e da pluralidade, critério ético pautado pela alteridade e pela integralidade humana.

Palavras-chave: Diversidades de gênero. Ética da alteridade. Formação acadêmica. Cultura jurídica.

\section{ABSTRACT}

The present article brings to the debate the importance of issues inherent to gender diversities being contemplated in the training of Law operators. We start from the confirmation of the exhausting of the juridical culture centered 
on adversarial values and practices, of maximizing individualistic gain and dehumanization of social-political relations. The study adds to the understanding that different knowledges are related to different customs, behaviors and praxis. Thus, transformations in the matrix of knowledges that form citizens have the potential to produce transformations in social relationships. An interdisciplinary theoretical referential, with contributions from Social Sciences, Law, Education and Philosophy, is adopted, in order to evaluate the inclusion of themes related to gender diversities as mandatory in pedagogical projects and syllabi of Law graduation courses in Porto Alegre, RS and its region, according to the current Human Rights Education policy. The concepts of conflict, politics, ethinic and otherness, in Jacques Rancière, liberalism, political rationality, transversal fights and knowledge-and-power in Michel Foucault are highlighted, to sustain the necessary transformation of the juridical culture and academic training. The individual potential is affirmed not as a good per se, but in order to promote the integration from the differences and plurality, an ethical criterion pervaded by otherness and human integrity.

Keywords: Gender diversity. Otherness ethics. Academic training. Juridical culture.

\section{Introdução}

$\mathrm{N}$

esse artigo busca-se contribuir para o debate, ainda aberto, acerca da importância de as questões inerentes às diversidades de gênero estarem contempladas na formação dos operadores do Direito nos cursos jurídicos brasileiros, partindo da constatação do exaurimento da cultura jurídica centrada nos valores e práticas adversariais de maximização do ganho individualista e desumanização das relações sócio-políticas. Neste artigo será abordada a ideia da ética da alteridade por meio da responsabilidade com o outro, e como essa perspectiva contribui para construir a adesão subjetiva à não violência e buscar a cultura da paz, admitindo-se a existência de um pluralismo jurídico, fomentando a necessidade da inclusão da disciplina de Mediação nas grades curriculares, tudo isso através da Educação.

Assim, sublinha-se a proposta de uma educação libertadora, capaz de desmistificar a ideia de que o educador é hierarquicamente superior aos educandos, de maneira que os dois aprendam juntos através da troca de experiências. Intenta-se compreender a importância da alteração da maneira como o Ensino Jurídico tem se baseado atualmente, qual seja, de modo essencialmente legalista. De modo que as transformações na matriz de conhecimentos que formam cidadãos têm potencial para produzir transformações nas relações sociais. Afirma-se, também, o potencial individual 
não como um bem em si, mas para que se promova a integração a partir das diferenças e da pluralidade, critério ético pautado pela alteridade e integralidade humana.

\section{A ética da alteridade para uma educação libertadora}

Quando se pensa em Ética da Alteridade, impreterivelmente, se faz necessário falar do filósofo Emmanuel Levinas, conhecido como o pensador da alteridade. Porém, cumpre salientar que em sua teoria, a alteridade ganha bastante visibilidade, mas não traz uma descrição fenomenológica em relação ao Outro, e sim sobre a reconstrução da subjetividade. É valido lembrar que a subjetividade foi uma das grandes novidades da filosofia moderna. Por meio dela uma vasta reflexão filosófica ocorreu, com a investigação do fenômeno do pensamento humano, intermediada pela subjetividade interpretada através do eu pensante.

Dessa maneira, Levinas vê a subjetividade através do Outro, onde não mais vale o modelo egoísta do Eu, que age de maneira individualista, e passa a prosperar um ambiente cortês e acolhedor. Diferentemente do modo que a subjetividade era encarada através da supervalorização do "Eu" como sujeito autônomo, o autor quebrou paradigmas em sua época e passou a pensá-la a partir da alteridade do Outro (MIRANDA, 2014).

Ainda explorando a ideia da subjetividade baseada na autonomia e liberdade, destaca-se o entendimento de Santos (1999), que traz a concepção de subjetividade aliada à cidadania. Segundo o referido autor, a relação entre cidadania e subjetividade é muito mais complicada do que parece, ao passo que ultrapassa a ideia rasa de liberdade e autonomia e abarca a questão da "autorreflexibilidade" - entendida como a reflexão de si próprio - da "autorresponsabilidade", que se compreende como a certeza de que cada pessoa é responsável por si mesma, da "materialidade de um corpo" e as peculiaridades que conferem à personalidade como algo único, exclusivo de cada ser humano.

Segundo Cibele Cheron (2014), a crise que estremece as estruturas da sociedade moderna é motivada pela supervalorização do individualismo que acaba por anular o outro, pela não tolerância à diversidade, pelo distanciamento do homem da política, pelo consumo exacerbado, dentre outras diversas características dos tempos atuais. Nesse contexto, surge a ideia de "ética da responsabilidade" ou "ética do diálogo", que tem como necessidade o consenso de vontades. Como preleciona Wolkmer (2001, p. 265): “A “ética da responsabilidade' nada mais é do que uma ética dialógica que se articula através da interação social, mediação que possibilita as 'condições de existência da Comunidade ideal com a Comunidade real". Em contrapartida, é de suma importância compreender que a sociedade não habita em um mundo perfeito, onde o homem vive livremente e age de forma consciente. Na realidade, esse sujeito que deveria ser compreendido e viver livremente é excluído e ignorado pela sociedade, por tal motivo que 
Wolkmer (2001, p. 268) propõe a chamada "ética concreta da alteridade", a qual visa à libertação e à emancipação dessa parcela oprimida da sociedade.

Segundo Miranda (2014), ao reestruturar a subjetividade, Levinas pretende elevar o homem a pensar sob uma nova perspectiva, onde esse é chamado à responsabilidade e ao acolhimento pelo Outro, ensejando a criação e um "novo humanismo", baseado na preocupação com o próximo. É importante salientar que essa responsabilidade a que Levinas se refere não se trata de uma vontade do sujeito que decide por sua conta assumi-la livremente, mas sim algo que vem antes até da sua própria liberdade, constituindo a subjetividade (MIRANDA, 2014).

De fato, é preciso inovar na forma do ensino, deixar de lado o modelo mecânico (a prática bancária do ensino, segundo Paulo Freire), onde o professor transmite algo, o aluno apenas reproduz uma cópia e não há uma reflexão sobre o que está sendo dito, tendendo à produção de profissionais sem consciência crítica, para dar lugar à educação libertadora, compreendendo-a não só como a transmissão do saber, mas como um processo de formação humana.

$\mathrm{Na}$ atualidade, as relações educacionais parecem estar sendo alicerçadas em modelos narrativos, onde há verdadeiros depósitos de conteúdos, o que acaba por conduzir a uma "memorização mecânica" do que está sendo transmitido pelo educador. Quanto mais se enche esse "depósito" dos educandos, melhor será a atuação do educador, conduzindo a educação a uma concepção "bancária" e a produzir educandos sem a devida consciência crítica.

Nesse modelo de educação não há transformação, não há vontade de fazer a diferença e a inquietação necessária para mudar o que não está bom. O educador é visto como um ser sábio que doa conteúdos àqueles que nada sabem. O educador libertador ou humanista é aquele que estimula o lado criativo e autêntico dos educandos, não o vê como um receptor de conteúdos, mas sim como um sujeito capaz de pensar por si mesmo e de raciocinar sobre aquilo que está ouvindo. Há uma profunda troca entre ambos, os dois aprendem juntos, e aí reside o lado humanista da educação libertadora. O educador não é aquele que apenas doa o que sabe, mas sim que encoraja o educando a perceber o seu poder criador.

A Educação como um processo humanista é capaz de ensejar aos educandos a vontade de querer fazer a diferença dentro da sociedade, uma vez que educação não é apenas o ato de transmitir o saber, mas sim de propiciar aos educandos um aprendizado de dentro para fora, através da conscientização desse de que ele é capaz de refletir sobre o que está ouvindo e de compreender o mundo de acordo com as suas próprias impressões. A proposta da educação libertadora está em desmistificar a ideia de que o educador é hierarquicamente superior aos educandos, de maneira que os dois sejam em conjunto educadores e educandos, e que através da troca de experiências ambos podem aprender. Nesta seara, os argumentos de autoridade não têm espaço.

Sendo através do diálogo que se encontra a essência da Educação, é através da palavra que se faz possível compreender o diálogo. Dessa maneira, para que haja uma verdadeira transformação do que está ao nosso redor é 
preciso entendê-la através da ação e da reflexão. A palavra deve estar acompanhada da ação, já que sem essa não há como haver transformação e, concomitantemente, da reflexão, pois, o agir sem observação dos possíveis resultados da conduta leva ao "verbalismo", ou seja, dá-se valor à palavra sem dar importância as suas reais ideias, o que acaba por transformar a palavra em algo sem utilidade, vazio, que não é capaz de produzir mudança, ensejando bloqueio do diálogo. Da mesma forma, não há como se falar em diálogo se esse é eivado de um ato prepotente, presunçoso, daquele sujeito que impõe o seu pensamento sem abrir espaço para que o outro aponte as suas ideias, a fim de que os dois evoluam juntos. Neste sentido, Paulo Freire (2013, p. 74) diz: "Como posso dialogar, se me fecho à contribuição dos outros, que jamais reconheço, e até me sinto ofendido com ela?". Desta maneira, é preciso humildade para haver diálogo, porquanto aquele se vê superior ao demais e não se abre à ideia de aprender com o outro não é capaz ensejar a verdadeira mudança do mundo. Apenas o diálogo está apto para produzir um pensamento crítico. Sem esse, não pode haver comunicação, tampouco Educação.

E, é por meio da Educação que fomentamos o sentimento de humanidade, sendo improvável pensarmos em uma cultura de paz sem que exista uma Educação voltada para esse sentido. A cultura da não violência nasce de "uma gama complexa de processos, entre eles processos educacionais, voltados para a promoção de relações pacíficas" (MILANI; JESUS, 2003, p. 198).

\section{O Direito e os cursos jurídicos como espaços androcêntricos}

Foi no final do século XVIII que localizamos as primeiras demandas femininas por direitos, consoantes às transformações profundas ocorridas nos campos social, político, econômico, científico, cultural e jurídico do mundo ocidental. Descobertas científicas, em especial no campo da anatomia, permitiram conhecer melhor o corpo humano e reconhecer as diferenças existentes entre os sexos. Por conta de tais diferenças anatômicas, pensadores iluministas defenderam a distinção radical entre homens e mulheres, associando atributos intelectuais e morais a caraterísticas físicas (FOUCAULT, 1977, 1984; SCHIEBNGER, 1987; LAQUEUR, 2001).

Um dos fundamentos teóricos das revoluções, que propiciaram o início à fase moderna do estado, por diversas vezes alicerçados em ideias de igualdade e liberdade para todos, possuem uma estrutura baseada em planos que não abarcaram a totalidade das diferenças entre as pessoas, acabando por excluir uma enorme parte de seus integrantes da sua condição de sujeito de direitos, devido a questões de raça, gênero ou expressão social (OLIVEIRA, 2002). Desenham-se lugares distintos para mulheres e para homens na ordem político-econômica dos estados capitalistas, afirmando a inferioridade das características e dos espaços destinados ao feminino. Dessa forma, "as atividades do homem eram dirigidas para o mundo social mais amplo da economia, política e interações sociais, além do âmbito 
da família, enquanto as de sua mulher eram rigidamente restringidas, limitavam-se ao mundo doméstico da própria família" (PARKER, 1991 p. 59).

O direcionamento epistêmico da modernidade valida apenas o conhecimento proveniente da lógica racional, instituída na dualidade natureza/homem ou incivilidade/civilidade. Trata-se de elementos opostos e dicotômicos, aos quais estão associadas as dicotomias corpo/razão e objeto/sujeito. Homem, civilidade, razão e sujeito estão adstritos ao masculino e, por sua vez, natureza, incivilidade, corpo e objeto estão conexos ao feminino. Por esse prisma, a epistemologia moderna constrói sujeitos masculinos neutros, que não necessitam de definição, à medida que o feminino é definido como o negativo do masculino. A mulher é o outro, que não é homem, definida pelo corpo, uma máquina incapaz da habilidade do pensamento: "tem ovários, um útero; eis as condições singulares que a encerram na sua subjetividade; diz-se de bom grado que ela pensa com suas glândulas". Impossível, nesse patamar, conceber que as mulheres possam ser sujeitos do conhecimento (BEAUVOIR, 2009, p. 16).

Verifica-se notório o predomínio masculino nas carreiras ligadas às profissões jurídicas, tanto que até 1960 foi mínima a participação das mulheres na advocacia. O Brasil, por sua vez, não se distanciou muito desse padrão. Estima-se que desde a formação dos cursos de Direito no País, em meados do século XIX, até a década de XX, tantos as carreiras públicas como as privadas foram ocupadas em sua maioria por "homens brancos ou embranquecidos pela posição social" (BONELLI, 2017, p. 96). Foi apenas na década de 1990, com o crescimento do número de cursos privado de Direito, através da ampliação do mercado do ensino jurídico, que as mulheres conseguiram disseminar a sua participação como profissionais. Tal participação perpassa pela mudança do modelo profissional que dominava até então, qual seja, aquele onde há predomínio masculino. Foi através da hibridização, ou seja, da mistura com as lógicas empresariais e organizacionais e com aumento das posições disponíveis que houve a diversificação do perfil dos docentes nos cursos de Direito (BONELLI, 2017).

A inclusão das mulheres na docência ganhou maior visibilidade na atualidade, porém ainda há predominância no chamado "ideário da neutralidade do conhecimento, construído por homens, brancos, heterossexuais, dos grupos estabelecidos, a partir de perspectivas eurocêntricas" (BONELLI, 2017, p. 98) E mais, os novos pontos de vistas elaborados por homens e mulheres, sendo esses brancos ou não, homoafetivos ou heterossexuais, dos grupos mais ou menos privilegiados, têm ajudado a desconstruir e "deslocar a produção canônica do conhecimento (BONELLI, 2017, p. 99). Para que seja possível fazer construir um novo paradigma do direito é preciso compreender a ideia de pluralismo jurídico, que de acordo com Wolkmer (2001, p. 219) pode ser compreendido como: "a multiplicidade de práticas jurídicas existentes num mesmo espaço sócio-político, interagidas por conflitos ou consensos, podendo ser ou não oficiais e tendo sua razão de ser nas necessidades existenciais, materiais e culturais". 
Para Wolkmer (2001), a teoria crítica pode ser definida como um mecanismo que permite ao sujeito estático a criação de uma consciência, o que ocasiona a construção de "agentes sociais" com uma visão do mundo mais comunicativa, revolucionária, transformadora. Por conseguinte, sendo a teoria crítica um "método" que advém das necessidades humanas essenciais, essa simboliza a criação de um novo paradigma no Direito, que, a priori, era reduzido a ir contra ao positivismo jurídico, porém passou a preocupar-se com a discussão dos "novos paradigmas éticos-jurídicos", que tinham em sua finalidade indagar como o Direito era ensinado nas faculdades, visando a novos meios de resolução de conflitos de forma "não repressiva".

É preciso que se tenha um pluralismo jurídico onde o seu suporte encontra-se na participação ativa da comunidade, devido à "insuficiência de resposta do Direito Tradicional às complexidades sociais" (WOLKMER, 2001, p. 219), propiciando um ambiente com menor incidência de desigualdades sociais, uma vez que a comunidade é chamada a falar sobre as suas mazelas. Entende-se que a democracia muda constantemente de referência. Admite-se, portanto, que haja a formação de novos direitos de maneira constante, assim, através da luta da sociedade há a concretização formal desses direitos. Por meio das lutas pela emancipação e pela igualdade houve a ampliação de diversos direitos, tais como: civis, políticos, sociais, da cidadania, das mulheres, crianças, idosos, sexuais e étnicos, também pelas lutas ecológicas e pelo meio ambiente sadio.

Uma importante diretriz do Plano Nacional de Ensino, intitulada "superação das desigualdades educacionais, com ênfase na promoção da igualdade racial, regional, de gênero e de orientação sexual" foi inserida por meio da PLC no 103/2012 e desde já sofreu críticas dos mais conservadores das igrejas católicas e evangélicas, em especial, as neopentencostais. A título de exemplo é válido mencionar uma nota da Conferência Nacional dos Bispos do Brasil (CNBB), emitindo um alerta à sociedade sobre os "perigos" da inclusão do que pode ser entendido como "ideologia de gênero" aos planos de Educação, informando que tal processo para inclusão da referida diretriz se deu de maneira silenciosa, sem perceber que o documento final da Conferência Nacional de Educação constituiu de modo democrático (FILHO; BORGES; ROBERTO, 2015).

A recusa do debate sobre a discussão de gênero e de sexualidades nos mais variados níveis da educação nacional é proveniente de um processo pedagógico direcionado para o controle das práticas sociais obtidas através da neutralização dos corpos por meio de uma visão binária dos gêneros, ou seja, só haveria a possibilidade da existência do sexo masculino e do feminino e esses, tidos como naturais. Se concebidos de uma maneira não natural, esses são vistos fora do padrão "heteronormativo" em questão. Assim, como se não bastasse excluir os corpos que não se adequam ao padrão estabelecido pela sociedade os homens são colocados em uma posição de superioridade em relação às mulheres, acabando por criar uma certa hierarquia entre os sexos, onde o papel atinente às mulheres atuavam de maneira subalterna, gerando uma sociedade andocentrista e heteronormativa (FILHO; BORGES; ROBERTO, 2015). 
De forma a não admitir fatos históricos que podem instigar uma quebra de confiança entre homens e mulheres, parece que a solução encontrada foi esconder o assunto, não o abordando nos cursos jurídicos. Porém, ao se ignorar essa questão, as novas gerações são educadas de forma parcial. Não aprendem que já houve um período de grandes desigualdades entre homens e mulheres, não conhecem a origem das lutas femininas por direitos, nem são estimuladas a refletir sobre as desigualdades que continuam a existir. Não aprendem que a luta por direitos é constante, embora fragmentada por refluxos e reviravoltas. Dessa forma, há um impedimento na educação para os direitos humanos, pois, ao ignorarem fatos do passado, as pessoas se tornam mais vulneráveis à repetição de situações de perda de direitos e retrocessos morais e legais, como observamos nos casos de refluxo de direitos.

A transversalização de gênero dentro do Direito, abrangida da graduação até cursos de capacitação, é uma importante e necessária ferramenta nas demandas que envolvam questões acerca das relações de gênero, porém, não é suficiente que tão somente o Direito, sendo esse norma ou ciência, legitime a categoria de gênero enquanto categoria de análise. É preciso ocorrer a mudança das relações de gênero em campos tidos como neutros, que agem de modo imparcial e avessos às influências externas, sendo esse o caso do Direito (SILVA, 2016).

Portanto, para que as mulheres não sejam postas em situações que acarretem prejuízo ou discriminação de maneira injustificada, com a desculpa do cumprimento dos rigores formais legais é preciso haver a adoção de uma postura "crítico-transformadora" em face dessas leis ou discursos. Assim, permite-se que haja superação das visões que justificam a manutenção da dominação masculina, como, por exemplo, nos casos de violência doméstica onde essas mulheres vitimadas por gravíssima violência, muitas vezes sexual, ainda assim continuam sendo tidas como causadoras ou provocadoras desse ato criminoso, mesmo estando "protegidas" por uma Constituição Federal considerada avançada e que possui em seu propósito, dentre outros, a construção da igualdade de gênero (SILVA, 2016).

O ensino jurídico, ao enfocar apenas os homens, esconde as mulheres que se destacaram na luta por direitos. Quando não se encontram modelos femininos de conduta jurídica, supõe-se que as mulheres não têm importância suficiente para que possam ser adotadas como modelo, forçando a adoção de modelos masculinos. Essa conduta apaga o passado de luta das mulheres, e o orgulho pela conquista de direitos. Cria-se, assim, um espaço determinado pelo poder masculino, no qual as mulheres são consideradas inferiores por não terem tido destaque no passado, da mesma forma que antigamente as mulheres foram consideradas homens imperfeitos e indignas de atenção. Sua consequência é insinuar que as mulheres são inadequadas para a vida pública e que, se elas quiserem ser uma referência, deverão se tornar homens. Como não podem modificar seus corpos, deverão incorporar as condutas masculinas no cotidiano, ignorando as suas características físicas e as suas especificidades biológicas e sociais. Essa desigualdade é agravada à medida que aumenta o número de mulheres ingressando nos 
cursos jurídicos, pois as obriga a se masculinizarem para serem aceitas nos bancos escolares, em uma repetição de um argumento antigo e restritivo das liberdades femininas. É nítida a violação do princípio da igualdade. A escola, ao ignorar o passado de conquistas femininas e glorificar apenas o histórico masculino, acentua uma desigualdade que conduz ao desrespeito à diversidade de gêneros e à segregação das mulheres não só no ambiente escolar, mas também na prática forense.

\section{Considerações finais}

$\mathrm{Na}$ análise foi possível perceber como a ética da alteridade, por intermédio da responsabilidade que cada um tem com o outro, pode contribuir de maneira otimista para a mudança de concepções baseadas no litígio, a fim de dar espaço à cultura de paz, através da admissão de que a sociedade está inserida em um espaço onde há pluralidade de práticas jurídicas, não mais se admitindo que ocorra o monopólio estatal de poder na tomada de decisões. Através da educação libertadora se faz possível desmistificar a ideia de que o educador é hierarquicamente superior aos educandos, uma vez que os dois têm a possibilidade de aprender juntos por meio da troca de experiências. Para que seja possível tal proposta, é necessário que o modelo mecânico de aprendizado, no qual o aluno apenas reproduz uma cópia do que o professor transmite sem a devida reflexão crítica do que está sendo dito, seja deixado de lado.

Fez-se uma reflexão acerca da importância do reconhecimento e da assistência permanente de políticas públicas que ajudem na efetivação dos direitos das mulheres, previstos na Constituição Federal, e da necessidade mais do que urgente de uma real mudança no sistema jurídico do Brasil, desde a formação dos operadores do Direito na graduação, até o quotidiano, onde essas leis são aplicadas, as quais possibilitam ou não a efetivação da emancipação das mulheres. Até porque, as políticas públicas, embora necessárias e importantes, ainda não são suficientes para garantir que as mulheres possam sentir e ver cumprir, no seu dia a dia, a igualdade de gênero. Daí a necessidade da modificação da forma como a educação jurídica é ministrada, deixando-se de lado, definitivamente, o ensino jurídico que glorifica apenas os homens, onde as mulheres são consideradas inferiores. Se faz necessária a busca incessante de outro modo de se conceber e de se consumar o Direito. Trata-se, por conseguinte, da necessidade de os futuros operadores do direito estarem preparados para fazer a diferença dentro da sociedade, cientes de que são capazes de transformar o que está a sua volta.

Acreditamos que uma significativa alteração pode ser fomentada com a inclusão da perspectiva não adversativa, através do ensino de mediação e outras dinâmicas autocompositivas. A mediação é um campo transdisciplinar, não se restringe a nenhum saber em especial e apresenta um conhecimento que é da humanidade: a habilidade em conduzir 
situações de conflito. Como aproveitaríamos a tecnologia da mediação judicial, estudando técnicas e aprimorando habilidades autocompositivas? Compor uma caixa de ferramentas interdisciplinar e atenta à complexidade do campo dos conflitos e às assimetrias sociais, como as calcadas no gênero e demais categorias em intersecção, é de importância estratégica. É inclusive por esse ponto, a dimensão emocional que, segundo Moore (1998), a mediação é geralmente definida como a interferência em uma negociação ou em um conflito de uma terceira parte. O mediador em questão, tem um poder de decisão limitado e não autoritário que ajuda as partes envolvidas a chegarem a um acordo mutuamente aceitável. Além de lidar com questões fundamentais, a mediação pode também estabelecer e fortalecer relacionamentos de confiança e respeito entre as partes ou encerrar relacionamentos de uma maneira que minimize os custos e os danos psicológicos.

A mediação caminha no sentido oposto à do conflito judicial, o qual origina um ganhador e um perdedor. A intervenção de um terceiro, o mediador, contribui, por meio da reabertura do diálogo, que a lógica experimentada na sessão seja a do ganha-ganha, justamente porque os sujeitos recuperam a sua autonomia para pensar, reconsiderar, negociar e resolver os seus impasses. Valoriza-se o poder da palavra e a posse dessa para cada um dos sujeitos envolvidos.

\section{Referências}

ALMEIDA, Frederico de Almeida; CAMARGO, Sarah Bria de; SOUZA, André Lucas Delgado. O Direito e a Realidade: desafios para o ensino jurídico. In: GHIRARDI, José Garcez; FEFERBAUM, Marina (org.). Ensino do direito em debate: reflexões a partir do 1 o Seminário Ensino Jurídico e Formação Docente. São Paulo: Direito GV, 2013. (Série Pesquisa Direito GV). Disponível em: http://bibliotecadigital.fgv.br/dspace/bitstream/handle/10438/11274/Ensino\%20do\%20 direito\%20em \%20debate.pdf?sequence=1. Acesso em: 20 maio 2017. https://doi.org/10.12660/rda.v261.2012.8861

BARBOSA, Águida Arruda. Mediação: Educar para Mediar. Revista Científica Virtual da Escolha Superior de Advocacia, [s. l.], n. 23, p. 33-43, 2016. ISSN 2175-4462. Disponível em: https://www.esaoabsp.edu.br/ckfinder/userfiles/files/RevistaVirtual/ Revista\%20Cienti\%CC\%81fic a\%20ESAOABSP\%20Ed\%2023.pdf. Acesso em: 10 out. 2016.

BONELLI, Maria da Gloria. Docência do Direito: fragmentação institucional, gênero e interseccionalidade. Cadernos de Pesquisa, [s. l.], v. 47, n. 163, p. 94-120, jan./mar. 2017. https://doi.org/10.1590/198053143659

BRAY, Renato Toller; GONZALEZ, Everaldo T. Quilici. Teoria Crítica no Direito e Pluralismo Jurídico: novos paradigmas éticos para o modelo jurídico nacional. Rev. de Direito da Unimep, [s. l.], v. 6, n. 11, p. 9-21, 2006. Disponível em: https://www. metodista.br/revistas/revistas-unimep/index.php/cd/article/view/645/214. Acesso em: 25 set. 2016. https://doi.org/10.15600/2238-1228/ cd.v6n11p9-21 
CARIELlO, Bruno; CARIELLO, Bruno; PEDRETE, Leonardo. Sociologia do direito: velhos e novos caminhos. Revista Habitus, Rio de Janeiro, v. 2, n. 1, p. 30-48, 30 mar. 2004. Disponível em: http://www.habitus.ifcs.ufrj.br/. Acesso em: 25 set. 2016. https://doi.org/10.34024/pensata.2018.v7.10104

CHERON, Cibele. Ética, autocomposição e empoderamento: um olhar sobre o manejo dos conflitos a partir de referencial endógeno. In: JORNADA DE MEDIAÇÃO DO TRIBUNAL DE JUSTIÇA DO RIO GRANDE DO SUL, 3., 2014, Porto Alegre. A mediação como marco transformador do Poder Judiciário. Anais [...]. Porto Alegre: NUPEMEC, 2014. https://doi.org/10.26668/indexlaw journals/2525-9679/2016.v2i2.1569

CUNHA, Djason B. Della Cunha. Pluralismo Jurídico e a ética do direito à alteridade. Revista do Instituto Brasileiro de Direitos Humanos, [s. l.], v. 2, n. 2, p. 73-83, 2001. Disponível em: http://www.corteidh.or.cr/tablas/r26066.pdf. Acesso em: 20 maio 2017.

FREIRE, Paulo. Pedagogia do Oprimido. Rio de Janeiro: Paz e Terra, 2013.

GAJARDONI, Fernando Fonseca. Novo CPC: vale apostar na conciliação/mediação? Jota Info, opinião e análise, 26 jan. 2015. Disponível em: http://jota.info/novo-cpc-vale-apostar-na-conciliacaomediacao. Acesso: 10 out. 2016.

GAIO JÚNIOR, Antônio Pereira; RIBEIRO, Wesllay Carlos. O Ensino Jurídico e os meios não contenciosos de solução de conflitos. R. Jurídica, Curitiba, n. 24, p. 13-25, 2010. Disponível em: http://www.gaiojr.adv.br/astherlab/uploads/arquivos/artigos/O_ ENSINO_JURIDICO_E_OS_MEI OS_NAO_CONTENCIOSOS_DE_SOLUCAO_DE_CONFLITOS.pdf. Acesso em: 15 ñov. 2016.

LEAL, Jackson da Silva; FAGUNDES, Lucas Machado. Pluralismo Jurídico e Justiça Comunitária na América Latina: potencialidades a partir da Sociologia das Ausências e das Emergências. Crítica Jurídica: Revista Latinoamericana de Política, Filosofía y Derecho, [s. l.], n. 35, p. 165-195, 2013.Disponível em: http://www.revistas.unam.mx/index.php/rcj/article/ view/40804/37133. Acesso em: 29 ago. 2016. https://doi.org/10.22201/ceichc.2013.40804

MILANI, Feizi M. JESUS, Rita de Cássia Dias P. (org.). Cultura de paz: estratégias, mapas e bússolas. Salvador: Edições INPAZ, 2003.

MIRANDA, José Valdinei Albuquerque de. Levinas e a reconstrução da subjetividade ética aproximações com o campo da educação. Rev. Bras. Educ., [s. l.], v. 19, n. 57, p. 461-475, 2014. ISSN 1413-2478. Disponível em: http://dx.doi.org/10.1590/ S1413-24782014000200010. Acesso em: 20 out. 2016. https://doi.org/10.1590/s1413-24782014000200010

PEREIRA, Clovis Brasil. Conciliação e Mediação no Novo CPC. [S. l.: s. n.]: 2015. Disponível em: http://www.conima.org.br/ arquivos/4682. Acesso em: 02 out. 2016.

ROSA, Conrado Paulino da. Desatando nós e criando laços: os novos desafios da mediação familiar. Belo Horizonte: Del Rey, 2012. 
SANTOS, Boaventura de Souza. Pela mão de Alice: o social e o político na pós-modernidade. 7. ed. Porto: Edições Afrontamento, 1994; São Paulo: Cortez, 1999.

SIDEKUM, Antônio. Emmanuel Levinas: educação e interpelação ética. Revista da FAEEBA - Educação e Contemporaneidade, Salvador, v. 39, p. 85-94. Disponível em: https:/www.revistas.uneb.br/index.php/faeeba/article/view/328/278. Acesso em: 20 maio 2017. https://doi.org/10.21879/faeeba2358-0194.v22.n39.328

SILVA, Érica Barbosa e. Profissionalização de Conciliadores e Mediadores. Revista Científica Virtual da Escolha Superior de Advocacia, ed. 23, p. 64-75, 2016, ISSN 2175-4462. Disponível em: https://www.esaoabsp.edu.br/ckfinder/userfiles/files/ RevistaVirtual/Revista\%20Cienti\%CC\%81fic a\%20ESAOABSP\%20Ed\%2023.pdf. Acesso em: 10 out. 2016.

WOLKMER, Antonio Carlos. Pluralismo Jurídico: fundamentos de uma nova cultura no direito. 3. ed. São Paulo: Alfa Ômega, 2001.

Recebido em: 17/12/2019.

Aprovado em: 20/12/2019.

Publicado em: 17/4/2020.

\section{Endereço para correspondência:}

Camila Paola Fernandes Polonia

Centro Universitário Ritter dos Reis

Rua Orfanatrófio, 555 - Alto Teresópolis

90840-440, Porto Alegre, RS, Brasil

\section{Autoras:}

Camila Paola Fernandes Polonia

Bacharel e Pós-Graduanda em Direito pela UniRitter Laureate Universities/ Centro Universitário Ritter dos Reis (UniRitter). Advogada.

Orcid: https://orcid.org/0000-0003-3422-0119

E-mail: c.fernandespaola@gmail.com

Cibele Cheron

Possui Bacharelado em Ciências Jurídicas e Sociais pela Universidade Federal do Rio Grande do Sul (2002), Mestrado em Ciências Sociais pela

Pontifícia Universidade Católica do Rio Grande do Sul (2006) e Doutorado em Ciência Política pela Universidade Federal do Rio Grande do Sul (2015).

É bolsista do Programa Nacional de Pós-Doutorado (PNPD/CAPES) junto ao Programa de Pós-Graduação em Educação da Pontifícia Universidade

Católica do Rio Grande do Sul. Bolsista PNPD/CAPES do PPGEDU/PUCRS.

Orcid: https://orcid.org/0000-0003-3501-5248

E-mail: iccibele@gmail.com

Endereço: Pontifícia Universidade Católica do Rio Grande do Sul

Av. Ipiranga, 6681, prédio 8, sala 304 - Partenon

97010-082, Porto Alegre, RS, Brasil 